\title{
Modélisation hydraulique du système Sebou-Fouarat, ville de Kenitra, Maroc - Cas des inondations de 2010
}

\author{
Abdellah Bourak, (MA) \\ Abdelbasset Midaoui, (MA) \\ Abderrahim Lahrach, (PhD) \\ Abdelkarim Elarrim, (PhD) \\ Abdel-Ali Chaouni, (PhD) \\ Laboratoire Géoressources et Environnement, \\ Université Sidi Mohamed Ben Abdellah, Fès, Maroc
}

doi: 10.19044/esj.2017.v13n12p368 URL:http://dx.doi.org/10.19044/esj.2017.v13n12p368

\begin{abstract}
The city of Kenitra, located in the extreme downstream of the Sebou basin, is threatened by floods caused by the overflowing of the Oued Sebou and the rise of Oued Fouarat. The hydraulic modeling study of the Sebou Fouarat system in transient mode is carried out by the HEC-RAS software and has implicated the two rivers. Two geometric models were constructed on the basis of a digital terrain model (DTM) using the Arc-GIS and HECGeoRAS softwares after processing the collected topographic data. The first model, of which the areas of Merja Fouarat and Al Assam have been represented by cross-section, is one-dimensional. The second one is also onedimensional in which the two areas of Merja Fouarat and Al Assam are introduced as water storage zones. The components of these models are the stream sections, lateral links, storage areas and junctions between the branches of each model. The flood hydrographs of the Sebou and Fouarat rivers are introduced as conditions at the upstream limits of the models while the tidal is introduced as a downstream condition. After the stability and calibration of the models, the results of the consulted hydraulic simulations are the variations of the water levels as well as the temporal variations of the flow rates for each section, the maximum flow velocities and the propagation times of the flood waves. The analysis and comparisons of these results strongly suggest using the second model for the treatment of the flood issues as a decision-making tool helping to manage floods during times of crisis.
\end{abstract}

Keywords: Hydraulic Modeling, HEC-Georas, Sebou, Fouarat, Kenitra 


\section{Résumé}

La ville de Kenitra, situé à l'aval extrême du bassin du Sebou, est menacée par les inondations émanant des débordements de l'oued Sebou et des crues de l'oued Fouarat. L'étude de modélisation hydraulique du système Sebou Fouarat en régime transitoire est réalisée par le logiciel HEC-RAS et a concerné ces deux oueds. Deux modèles géométriques ont été construits sur la base d'un model numérique de terrain par le bais des logiciels Arc-Gis et HEC-GeoRAS et après avoir traité des données topographiques collectées. Le premier modèle, dont les zones de la Merja Fouarat et d'Al Assam ont été représentées par des profiles en travers, est unidimensionnel. Le deuxième est également unidimensionnel dans lequel les deux zones de la Merja Fouarat et d'Al Assam sont introduites comme zones de stockage des eaux. Les composantes de ces modèles sont les tronçons du cours d'eau, les liens latéraux, les zones de stockage ainsi que des jonctions entre les branches de chaque modèle. Les hydrogrammes de crues des oueds Sebou et Fouarat sont introduits comme conditions aux limites amont des modèles alors que le marégramme est introduit comme condition aval. Après la stabilité et calibration des modèles, les résultats des simulations hydrauliques consultées sont les variations des niveaux d'eau ainsi que les variations temporelles des débits pour chaque section, les vitesses maximales d'écoulement et les temps de propagation des ondes de crues. L'analyse et les comparaisons de ces résultats suggèrent fortement l'utilisation du second modèle pour le traitement des problèmes d'inondation comme un outil d'aide à la prise de décision pour gérer les inondations en période de crise.

Mots-clés : Modélisation hydraulique, HEC-GeoRAS, Sebou, Fouarat, Kenitra

\section{Introduction}

La zone de Fouarat et d'Al Assam, située dans la ville de Kenitra, se trouve sur la côte atlantique marocaine à $40 \mathrm{~km}$ au nord de la ville de Rabat (fig. 1).

Cette étude s'intéresse à l'analyse des écoulements du système hydraulique 'Sebou-Fouarat'. Ce système est formé par le tronçon aval de l'oued Sebou allant de l'autoroute à l'ouest de la ville Kenitra jusqu'à son embouchure et aux niveaux de la zone Al Assam située sur sa rive gauche, la zone de la Merja et du canal Fouarat et les connexions entre ces entités. Ce système hydraulique est à l'origine de sérieuses inondations par l'extension des plans d'eau en périodes de fortes pluies ce qui touche ainsi les populations et leurs bien ainsi que les infrastructures de base situées dans l'enceinte de cette zone. Pour remédier à ces inondations, une modélisation 
hydraulique sera établie en vue de simuler la réponse de ce système à la crue vécue en 2010 et d'en tirer les conclusions pour lutter contre les effets des inondations.

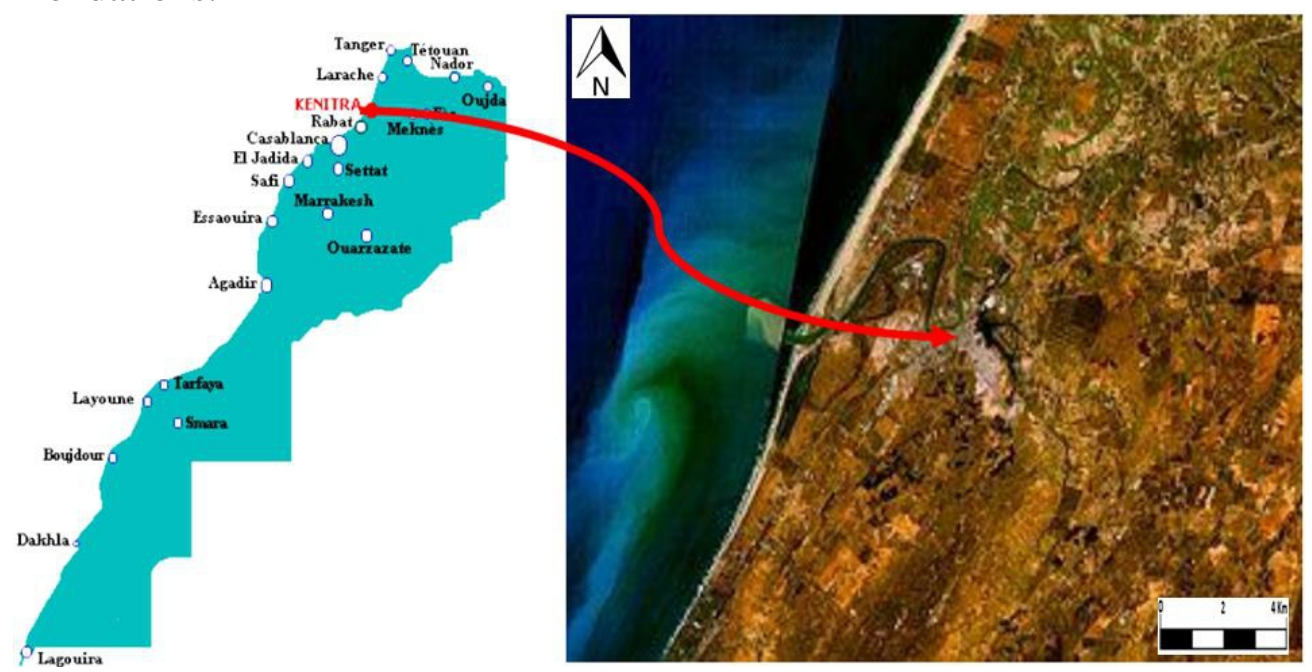

Figure 27 : Situation de la zone d'étude

\section{Données générales de la zone d'étude}

La zone est constituée des régions basses de la rive gauche de l'oued Sebou et de la Merja Al Fouarat; De point de vue topographique, la plus grande partie est située entre les côtes de 1 et $9 \mathrm{~m}$ NGM. Elle est limitée à l'Est et au Nord par les berges de l'oued Sebou.

Faisant partie de la zone du Gharb, le climat de cette zone est de type méditerranéen à influence océanique, il est marqué par deux grandes saisons qui sont l'hiver et l'été caractérisés simultanément par le déplacement d'une masse d'aire polaire et de la montée en été d'une masse d'aire tropical, le tout étant relié à la position relative de la zone anticyclonique des Açores.

D'après la classification bioclimatique d'Emberger, elle appartient à l'étage subhumide à hiver tempéré sur la frange côtière. La pluie moyenne annuelle de la ville de Kenitra est de $555 \mathrm{~mm}$ sur les 33 années passées (fig.2).

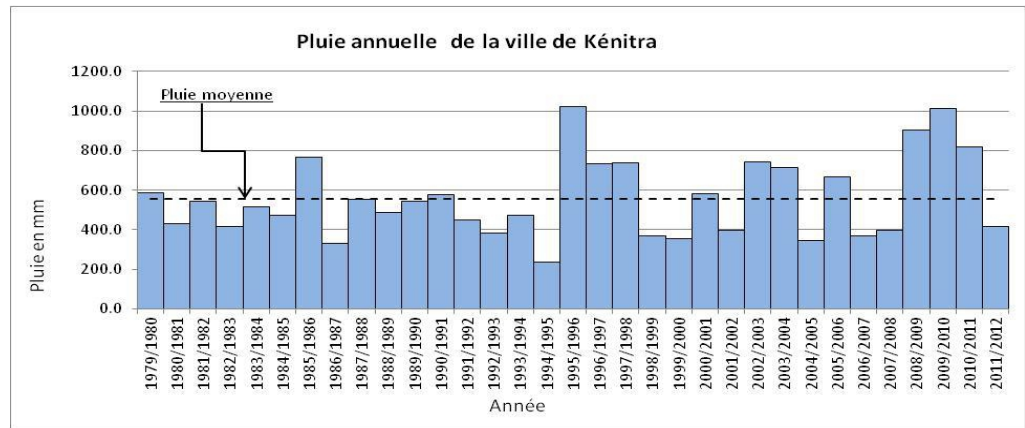

Figure 2 : Pluie annuelle du poste de Kenitra - (ABHS, 2013) 
Ces précipitations sont caractérisées par une grande variabilité interannuelle avec un coefficient de variation allant jusqu'à $140 \%$. Pour la distribution mensuelle des précipitations, la période pluvieuse se situe entre les mois d'octobre et le mois de mai, cette période reçoit plus de $96 \%$ de la quantité annuelle. La température moyenne annuelle varie entre 12.8 et 22.4 ${ }^{\circ} \mathrm{C}$

La géomorphologie de cette zone en forme de cuvette subsidente, fermée vers la mer par le sahel dunaire et le caractère imperméable des formations à limons et sols noirs argileux (tirs) lui confèrent un caractère endoréique. Les écoulements se concentrent en effet, au niveau de ces zones et forment des Merjats comme celles qui se trouvent en aval de l'oued Al Fouarat et celui d'Al Assam.

\section{Position du problème, objectif et approche de l'étude}

\subsection{Problématique des inondations dans la ville de Kenitra}

Les inondations de la ville de Kenitra, fréquemment observées au cours des périodes pluvieuses, sont liées principalement à deux sources différentes, il s'agit principalement des débordements des eaux de l'oued Sebou qui présente une capacité de transit très faible au niveau de son passage dans la ville et secondairement aux crues des bassins de l'oued Fouarat (Lahrouz, 2011). Les inondations les plus marquées dans les dernières trentaines d'années sont celles observées en 1996, en 2009 et en 2010 et qui ont été enregistrées dans plusieurs quartiers de forte densité et des infrastructures de base.

\subsection{Objectif de l'étude}

L'objectif de l'étude est de comprendre, d'analyser et d'interpréter le comportement et le régime du système hydraulique Sebou-Fouarat par le biais de deux modèles géométriques unidimensionnels différents selon qu'ils prennent ou non en considération, en plus des écoulements dans les oueds et dans les couloirs préférentiels, de la capacité de stockage et de laminage offertes par les dépressions topographiques (Storage area).

Et enfin, selon les résultats, recommander le modèle le plus approprié pour traiter la problématique des inondations que ce soit l'aspect structurel définissant les mesures de protection contre les inondations ou non structurel permettant la délimitation des zones inondables à prendre en considération dans l'aménagement du territoire (Barroca, 2008).

\subsection{Approche et méthodologie adoptées}

L'approche adoptée dans cette étude est scindée en trois parties: (i) la collecte des informations disponibles et les investigations de terrains en particulier la recherche des traces de crues, le recueil des témoignages sur les 
événements historiques vécus ainsi que leur synthèse et leur analyse; (ii) la modélisation hydraulique pour les deux modèles géométriques; (iii) l'analyse et l'interprétation des résultats en vue de tirer les conclusions et les recommandations sur l'aspect aménagement hydraulique de cette zone.

Apres avoir analysé toutes les informations collectées y compris les donnes topographique et hydrologiques relatives aux débits de crues, deux modèles hydrauliques sont construits à l'aide des modèles HEC-RAS et HEC-GeoRAS (USA.IWR, 2001).

Pour simuler le fonctionnement hydraulique du complexe Sebou Fouarat, les deux modèles développés sont:

- Le premier modèle «modèle 1 » est un modèle unidimensionnel (1D) et prend en considération les zones de la Merja Fouarat et d'Al Assam comme des tronçons de cours d'eau, les branches des cours d'eau, les jonctions, les déversoirs latéraux (fig.3).

- Le deuxième modèle "modèle 2 » quant à lui est aussi un modèle unidimensionnel (1D), il comprend en plus des composantes du Modèle 1, les zones de stockage (Storage area) et leurs connexions (fig.4)

Les deux schémas représentatifs des modèles géométriques à étudier sont comme suit :

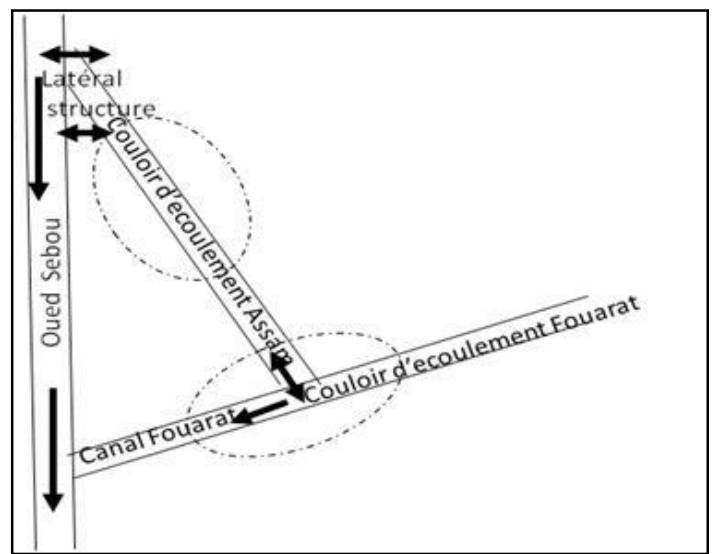

Figure 3 : Modèle unidimensionnel (1D) « Modèle 1 »

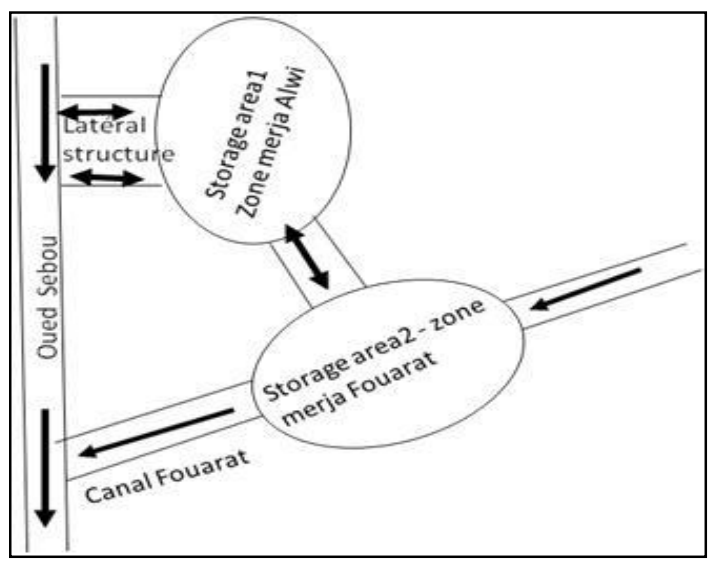

Figure 4 : Modèle unidimensionnel (1D) avec zones de stockages « Modèle 2 »

Une fois les modèles géométriques sont établis, ils sont alimentés avec les données relatives aux conditions aux limites, les conditions initiales à l'amont et à l'aval. Après la correction des erreurs, de stabilité et de calibration des modèles, les résultats sont visualisés pour effectuer les différentes analyses et comparaisons possibles (fig.5). 


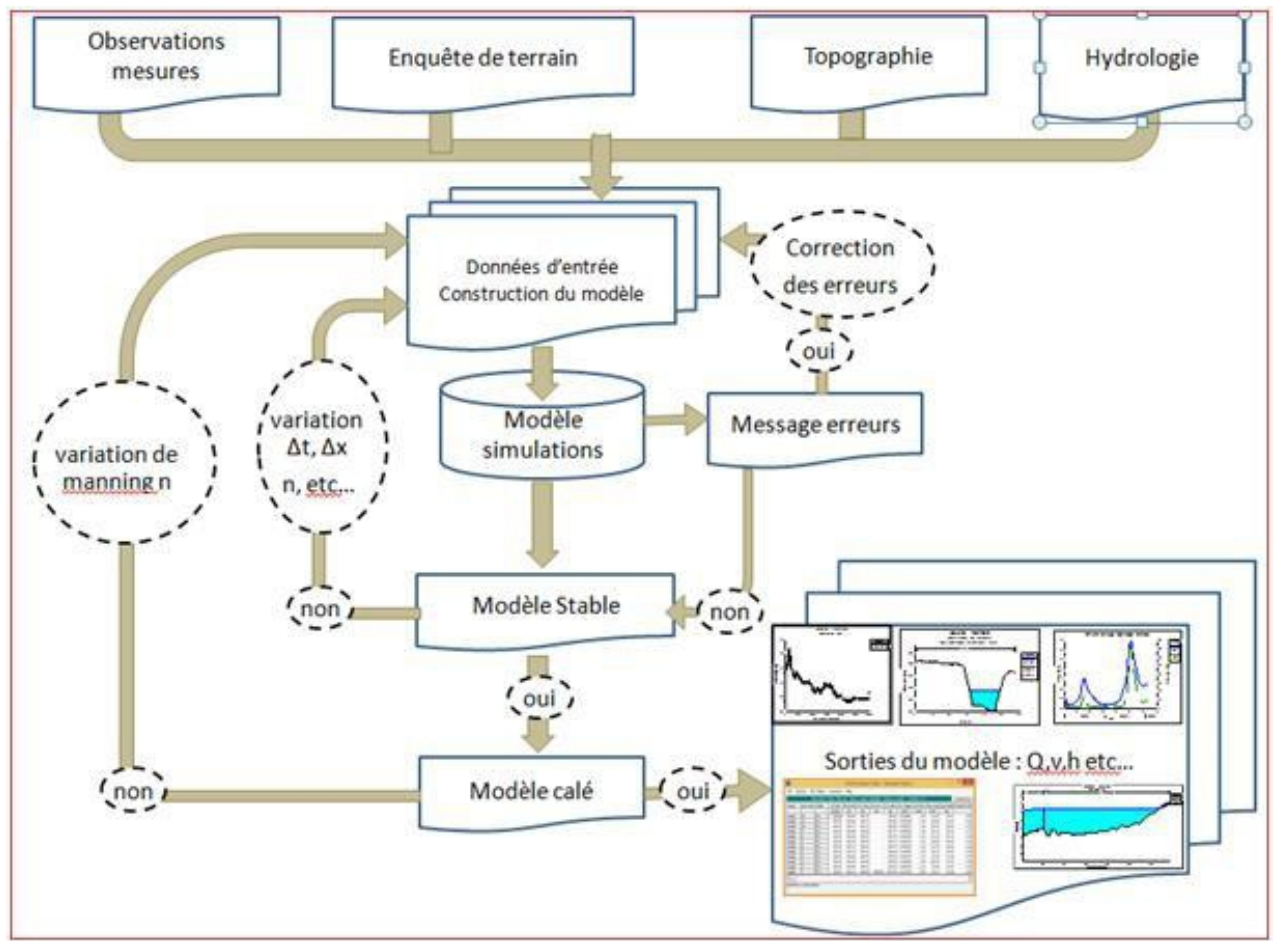

Figure 5 : Schéma des modèles hydrauliques

L'analyse a porté sur les résultats hydrauliques pour les deux modèles conceptuels, pour la crue de 2010 de l'oued Sebou et l'hydrogramme de la crue centennale de l'oued Fouarat en l'occurrence les vitesses, les niveaux d'eau maximums atteints, les propagations des ondes de crues. Cette analyse sera faite à partir des simulations hydrauliques du système Sebou Fouarat à l'aide du modèle HEC-RAS. Et enfin, pour recommander le modèle le plus adéquat pour étudier cette problématique, une analyse des résultats issus des deux modèles a été faite pour explique les divergences qui y sont constatées.

\section{Analyse et diagnostic du système hydraulique Sebou Fouarat}

Suite aux différentes études et investigations menées sur les lieux et aux différentes analyse des documents collectés en relation avec le thème, la description des inondations causées par l'oued Sebou et l'oued Fouarat et ses affluents sera abordée dans cette analyse séparément. En effet, lors des crues de 2010, les débordements de l'oued Sebou sont observés juste à l'amont de la zone Assam, au niveau du bras mort de l'oued situé sur sa rive gauche. Par la suite, ces eaux transitent vers la Merja Al Fouarat, en empruntant l'ancien axe de son écoulement (fig.6). Les cotes observées selon les témoignages ont dépassé 4 m NGM dans quelques endroits de la zone. 


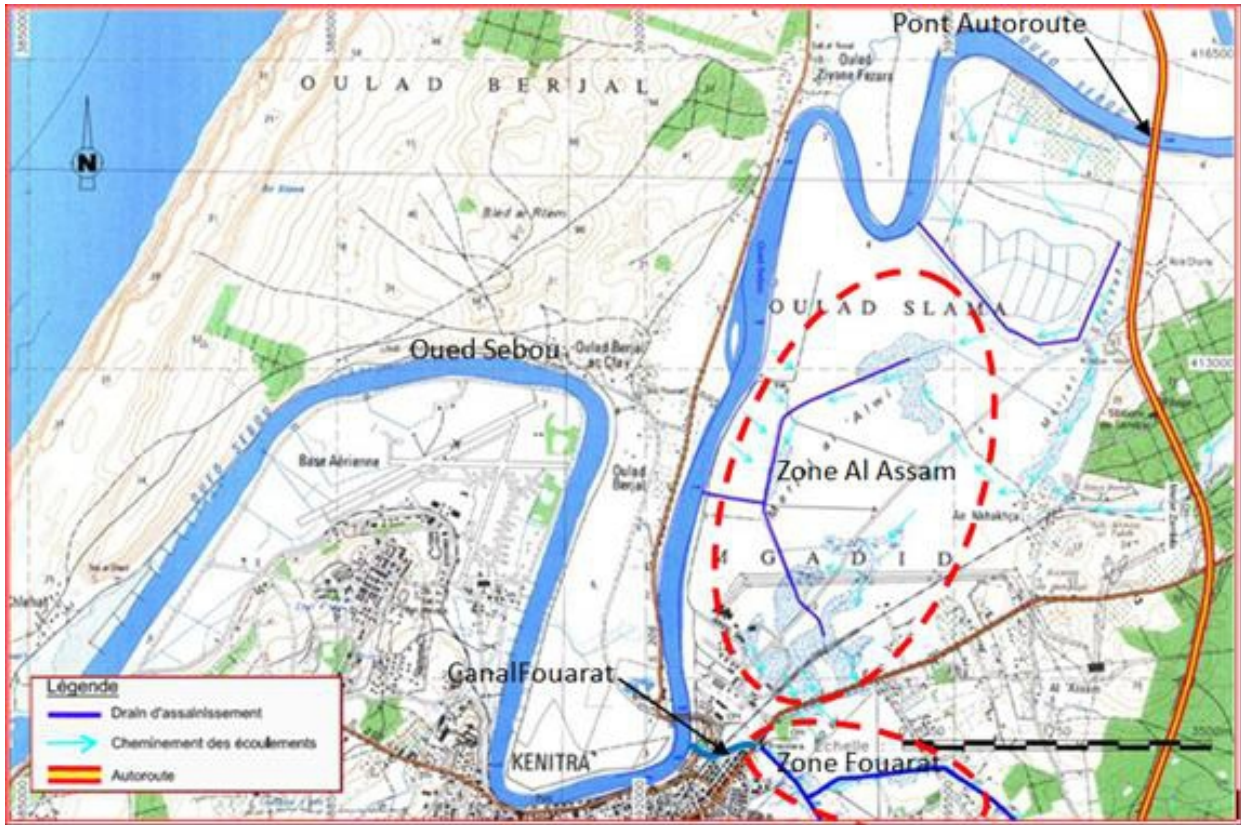

Figure 6 : Carte du système hydraulique Sebou Fouarat

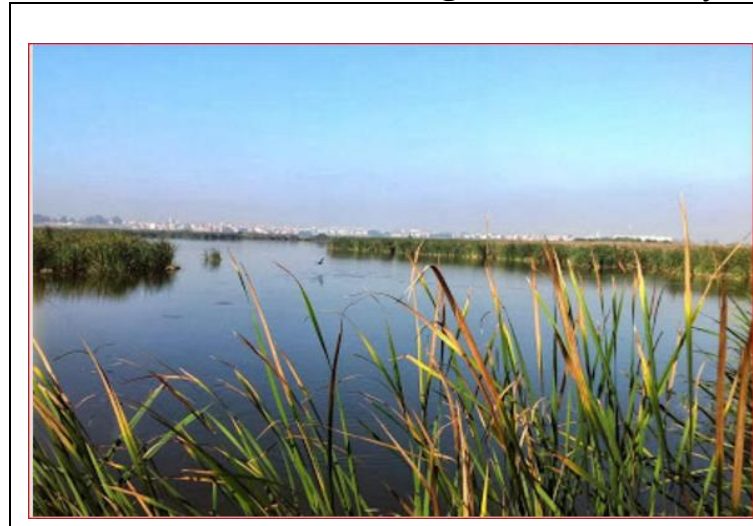

Figure 7 : Vue amont de la Merja Fouarat

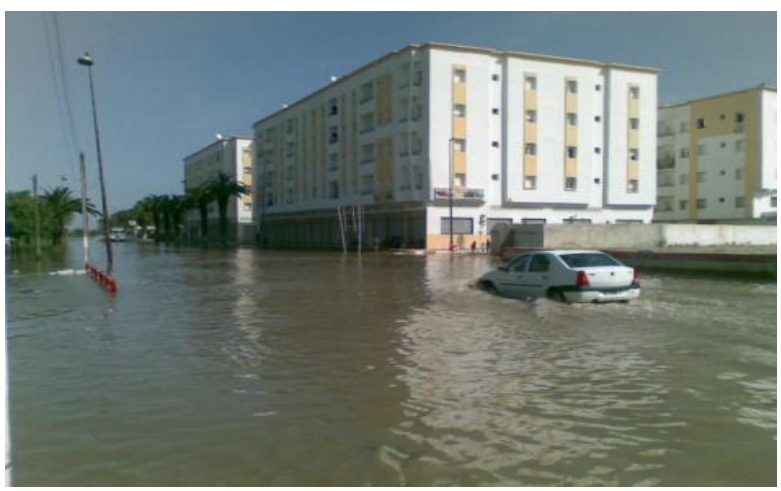

Figure 8 : Inondation au niveau de la ville de Kenitra en 2010

\section{Simulation hydraulique du système Fouarat-Sebou}

\subsection{Analyse des données hydrologiques et de la marée}

Les données hydrologiques sur les hydrogrammes de crues utilisées et les conditions aux limites dans les simulations hydrauliques sont :

- Branche Oued Sebou : L'hydrogramme de la crue de Janvier à Mars de l'année 2010 (fig.9) issu de l'étude du schéma directeur de protection de la plaine du Gharb contre les inondations qui tiennent compte de la marée (les petites oscillations sont dues à l'impact de la marée) 
- Branche Fouarat: L'hydrogramme de la crue centennale (fig.10) utilisé est celui élaboré dans le cadre de l'étude de protection de la ville de Kenitra contre les inondations. Cet hydrogramme est pris à l'entrée de la branche Fouarat

- Les données du marégramme (fig.11) sont également introduites dans le modèle comme condition imposée à l'aval de l'oued Sebou.

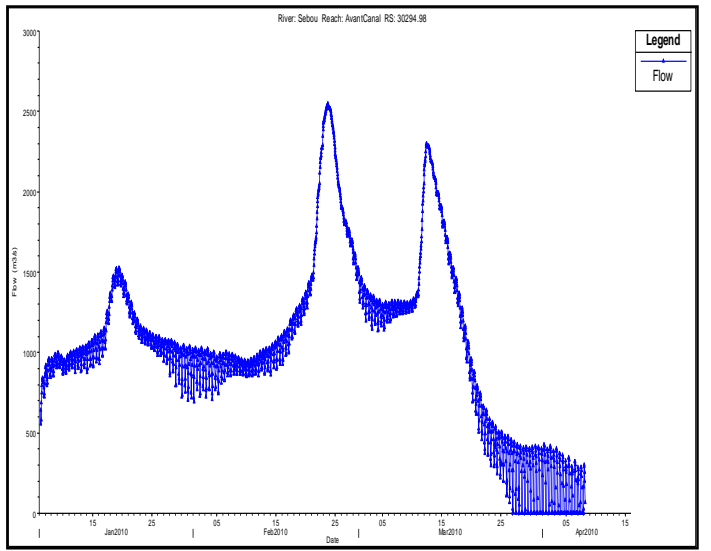

Figure 9 : Hydrogrammes de crue de l'oued Sebou (ABHS, 2010)

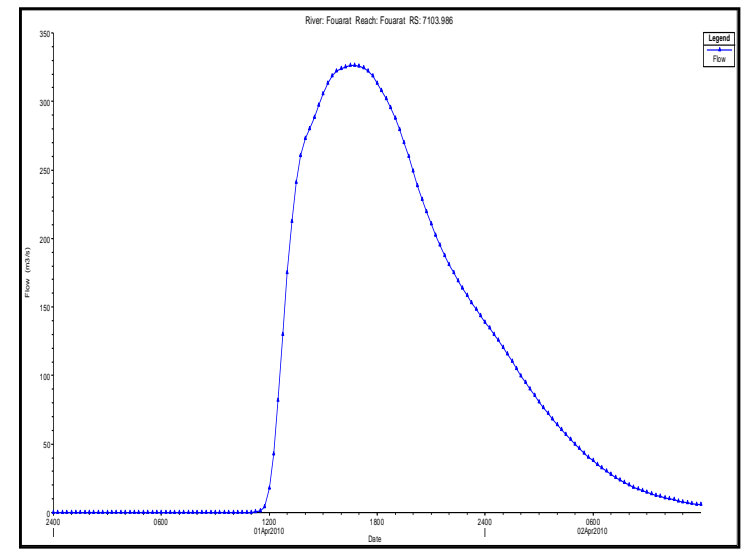

Figure10 : Hydrogrammes de crue de l'oued Sebou (ABHS, 2013)

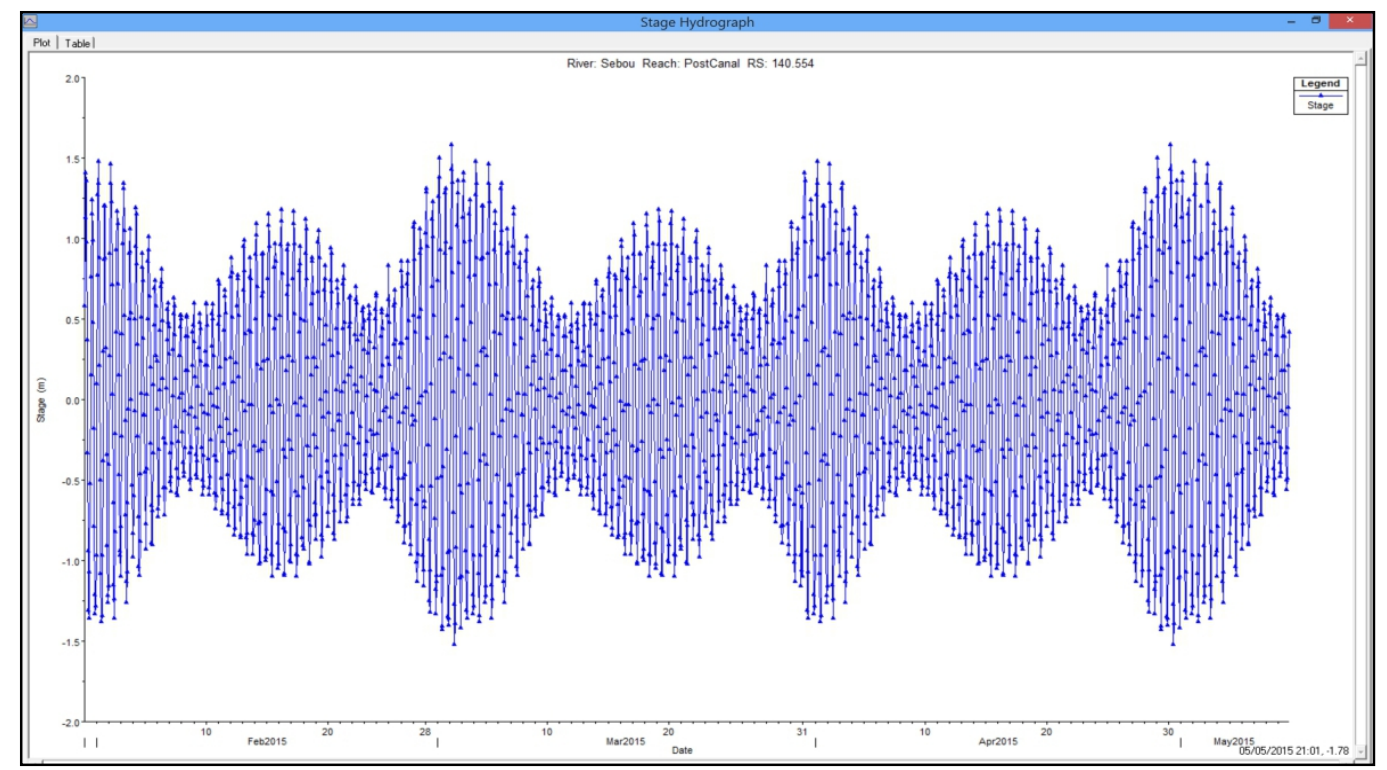

Figure 11 : Marégramme au niveau du port de Kenitra, (ABHS, 2010)

\subsection{Analyse des données topographiques}

Les documents topographiques collectés sont : 
- Des profils en travers et bathymétriques de l'oued Sebou et du canal et de la Merja Fouarat ;

- Des plans côtés des digues existantes et de la zone des deux Merjas, de la route nationale, de la voie ferrée;

- Des restitutions numériques de la ville de Kenitra.

- Apres la vérification et l'analyse des données topographiques en se basant sur les investigations effectuées sur le terrain et sur une analyse comparative des différentes données collectées un modèle numérique de terrain a été établi par ARCGIS pour le système Sebou- Fouarat (fig.12).

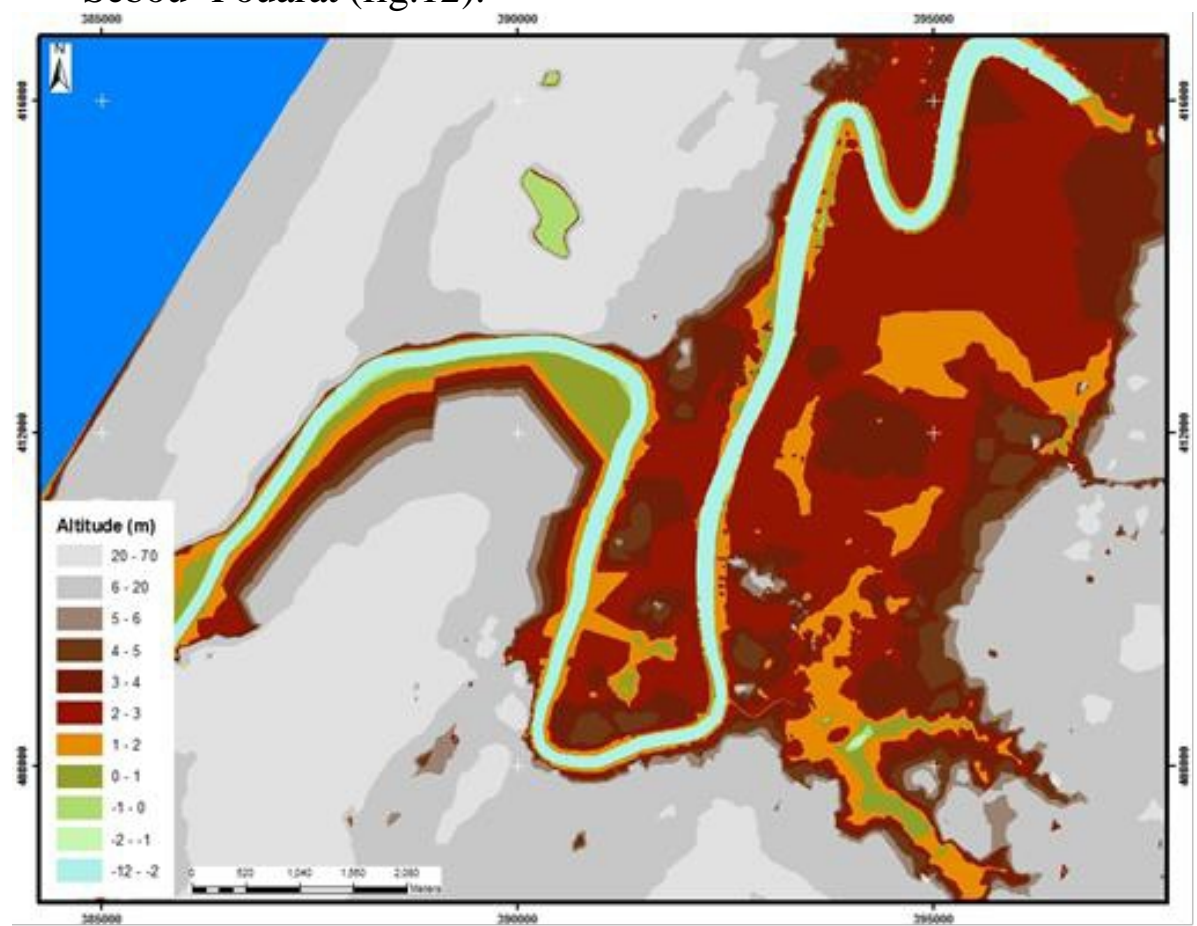

Figure12 : Modèle numérique de terrain de la zone d'étude

\subsection{Modèles géométriques}

Sur la base du modèle numérique du terrain, deux modèles géométriques ont été conçus par l'utilitaire HEC-GeoRAS pour étudier la réponse hydraulique du système Sebou-Fouarat aux crues dans cette zone.

\subsubsection{Modèle 1}

Ce modèle (fig. 13) est composé de :

- Six branches : «Tronçon Sebou Amont», «Alwin Amont», «Alwi Aval », «Alwi Sebou », «tronçon Sebou aval » et « Tronçon Canal Fouarat $»$; 
- Trois jonctions qui permettent de modéliser les échanges continues entre le tronçon amont de oued Sebou et la zone d'Al Assam (Merja Alwi) ;

- Deux jonctions : «Fouarat-Canal Fouarat-Alwi» et «Canal FouaratTronçon Aval Sebou-Tronçon Amont Sebou »;

\subsubsection{Modèle 2}

Ce modèle (fig. 14) est composé de :

- Trois branches : «Tronçon Sebou Amont », «Tronçon Sebou Aval » et « Tronçon canal Fouarat »;

- Deux liens latéraux qui permettent de modéliser les échanges entre le tronçon amont de l'oued Sebou et de la zone Al Assam (Lateral Stuctures) ;

- Deux zones de stockage qui sont la Merja Fouarat et la zone Al Assam ou Merja Alwin (Storage Area);

- Trois jonctions : «Alwin-Fouarat », «Fouarat-Canal Fouarat » et «Canal Fouarat-Tronçon Aval Sebou-tronçon amont Sebou »

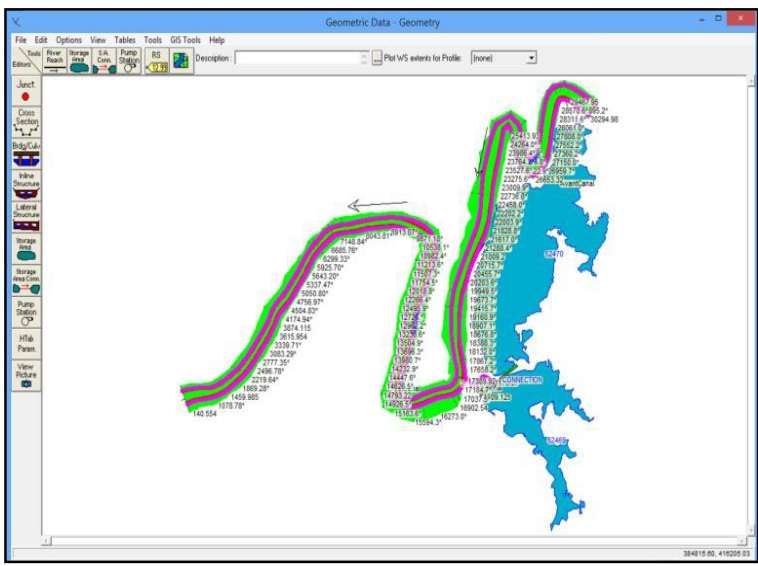

Figure 13 : Modèle Sebou-Fouarat unidimensionnel avec Storage area (Modèle 2)

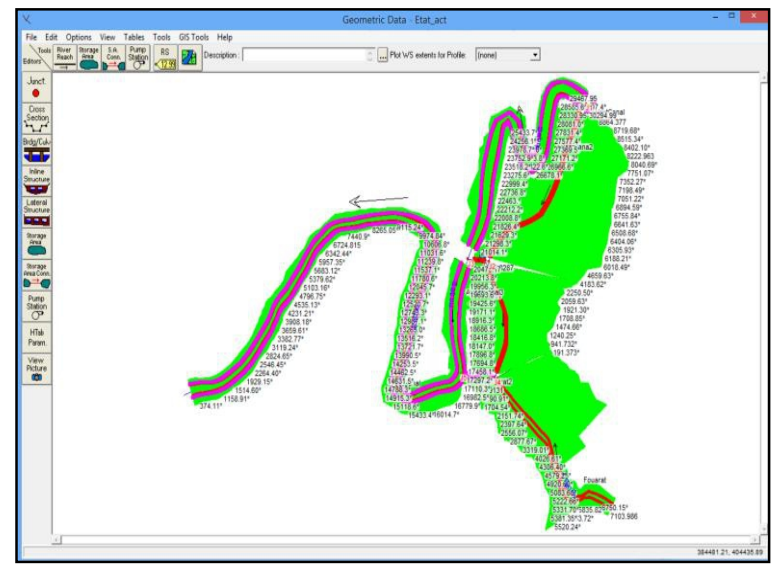

Figure 14 : Modèle Sebou-Fouarat unidimensionnel (Modèle 1)

\subsection{Simulations hydrauliques et calibration}

Apres avoir procédé aux corrections des erreurs et à la stabilisation des deux modèles par la vérification des données, les ajouts des profils en travers par interpolation et des variations du pas temps de simulations, plusieurs itérations pour des coefficients de Manning ont été menées pour calibrer les modèles.

Faute d'un historique précis des niveaux d'eau atteints lors du passage de la crue de 2010, les repères de certaines laisses de crues et l'étendue de la zone inondée ont été utilisées pour caler les deux modèles. Ce calage est fait 
par comparaison entre des niveaux d'eau résultant des simulations hydrauliques par différents coefficients de Manning avec les niveaux d'eau maxima observés au niveau de deux points de contrôle (Malaterre, 2011) (tab.1).

Tableau 1 : Comparaison des niveaux d'eau maxima simulés et observés

\begin{tabular}{|c|c|c|c|c|c|}
\hline $\begin{array}{c}\text { Points de } \\
\text { contrôle }\end{array}$ & $\begin{array}{c}\text { Niveau } \\
\text { observé en } \\
\text { mNGM }\end{array}$ & $\begin{array}{c}\text { Niveau calculé } \\
\text { en mNGM - } \\
\text { Model 1 }\end{array}$ & $\begin{array}{c}\text { Niveau calculé } \\
\text { en mNGM - } \\
\text { Model 2 }\end{array}$ & $\begin{array}{c}\text { Erreur } \\
(\%) \\
\text { Model 1 }\end{array}$ & $\begin{array}{c}\text { Erreur } \\
(\%) \\
\text { Model 2 }\end{array}$ \\
\hline Zone Assam & 5.2 & 6.2 & 4.8 & $19 \%$ & $-6 \%$ \\
\hline Port de Kenitra & 3.3 & 3.5 & 3.2 & $6 \%$ & $-3 \%$ \\
\hline
\end{tabular}

\section{Présentation et discussion des résultats hydrauliques}

Après les simulations hydrauliques qui se sont basées sur la concomitance de la crue de janvier à Mars de l'année de 2010 de l'oued Sebou et de la crue centennale des bassins versants de l'oued Fouarat selon les modèles de simulation d'écoulement transitoire (Kovacs, 2010), les résultats concernent les hydrogrammes de crues, les niveaux maxima des plus hautes eaux, les vitesses maximales sont consultés pour effectuer les différentes comparaison et analyses.

Sachant que le débit maximum de l'hydrogramme injecté en amont de la zone Assam dans l'oued Sebou est de l'ordre de $2550 \mathrm{~m}^{3} / \mathrm{s}$ (fig.9), les débits maxima débordant sur la zone Assam sont de l'ordre de $900 \mathrm{~m}^{3} / \mathrm{s}$ (fig.17) pour le modèle 1 et de $750 \mathrm{~m}^{3} / \mathrm{s}$ (fig.16) pour le modèle 2, alors que les débits restants dans l'oued Sebou sont respectivement de $1700 \mathrm{~m}^{3} / \mathrm{s}$ (fig.15) et de $1900 \mathrm{~m}^{3} / \mathrm{s}$ (fig.16). Il a été constaté également que le débit maximum retourné vers d'oued Sebou de la zone Assam sont est de $450 \mathrm{~m}^{3} / \mathrm{s}$ (fig.19) pour le modèle 1 et de $750 \mathrm{~m}^{3} / \mathrm{s}$ (fig.20) pour le modèle 2 .

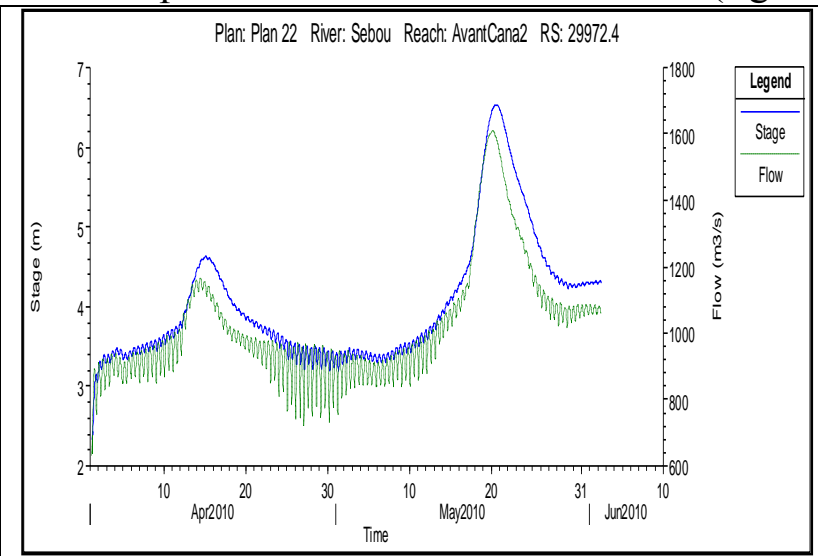

Figure 15 : Hydrogramme et niveau d'eau dans l'oued

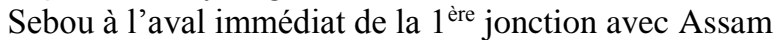
Modèle 1

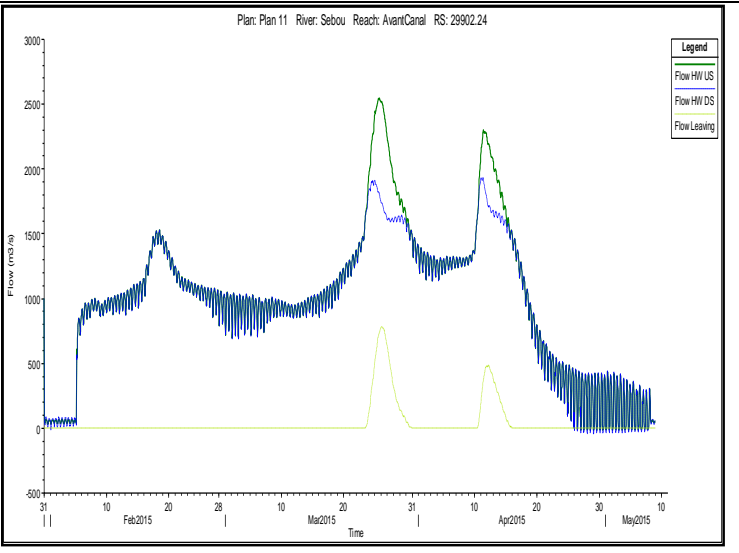

Figure 16 : Hydrogrammes au niveau du lien latérale amont Sebou Assam - Modèle 2 


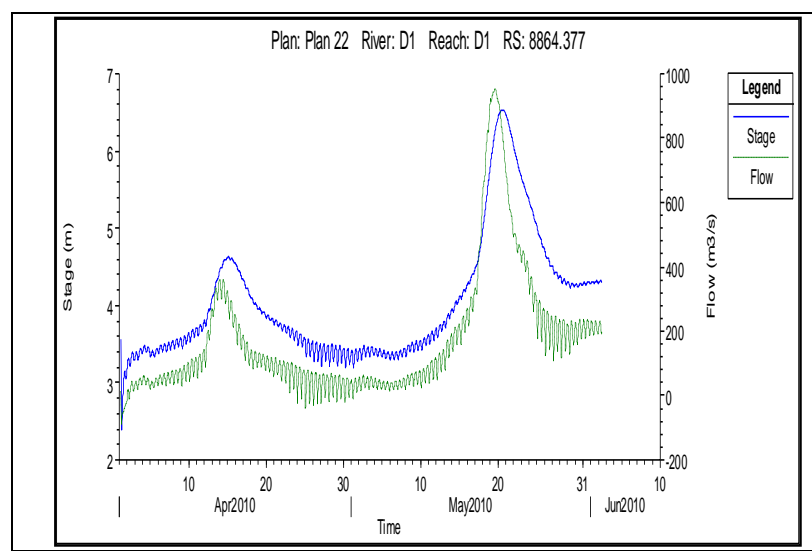

Figure 17 : Hydrogramme et niveau d'eau dans la 1 ère jonction avec Assam Modèle 1

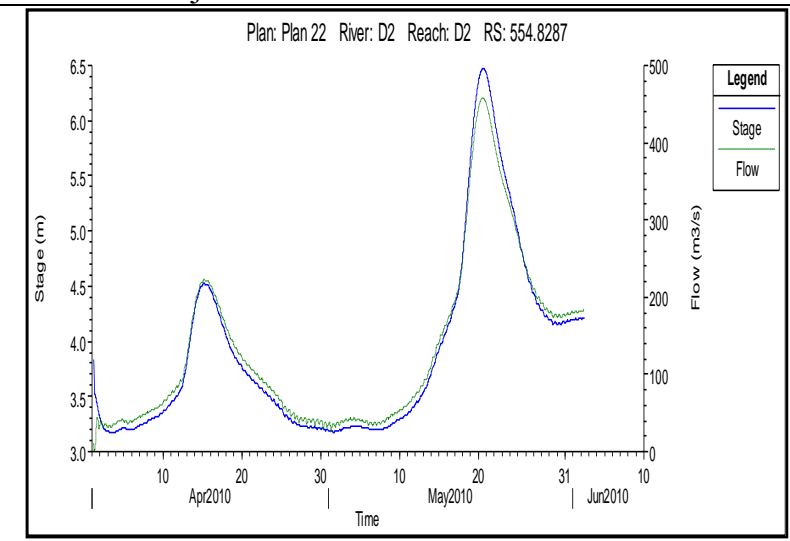

Figure 19 : Hydrogramme et niveau d'eau dans la $2^{\text {ème }}$ jonction avec Assam Modèle 1

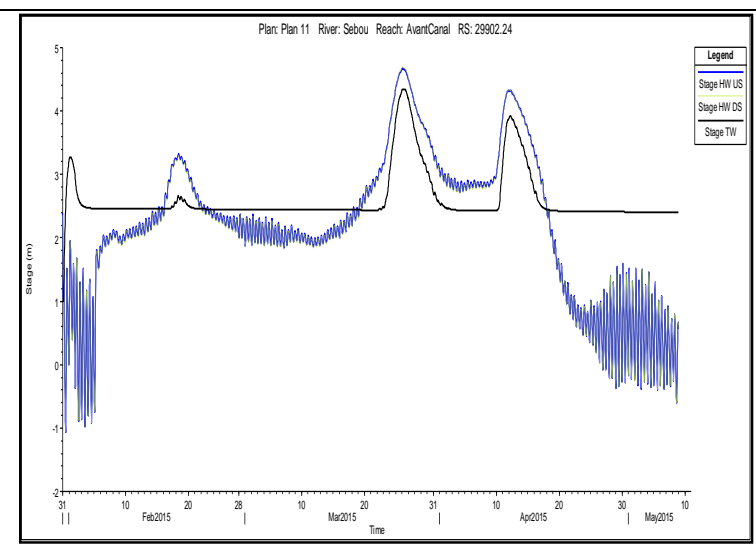

Figure 18 : Niveaux d'eau du lien latérale Sebou Assam Modèle 2

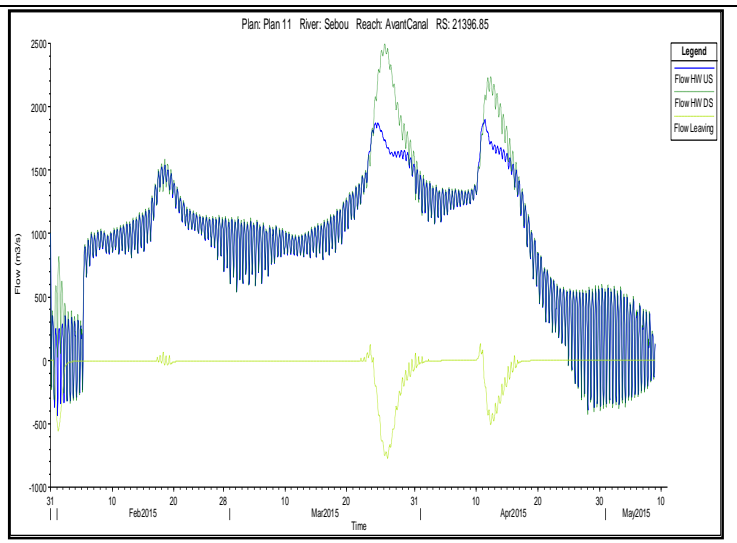

Figure 20 : Hydrogrammes au niveau du lien latéral aval Sebou Assam - Modèle 2

Les différences observées dans les débits simulés par les deux modèles au niveau du premier point de débordement sont dues à la capacité de stockage introduite dans le $2^{\text {ème }}$ modèle qui a réduit les débits a l'aval et favorisé les conditions d'écoulement de l'amont vers l'aval dans l'oued Sebou. Pour les modèles, l'effet de la crue des bassins de Fouarat n'a pas été ressenti au niveau d'Al Assam puisque il reste négligeable de point de vue débit et volume. En effet, pour le cas du modèle 2, l'hydrogramme de Fouarat présente un débit de pointe de $325 \mathrm{~m}^{3} / \mathrm{s}$ et un temps de base de 24 heures, alors que celui émanant des débordements du Sebou a deux pics l'un à $800 \mathrm{~m}^{3} / \mathrm{s}$ et l'autre à $500 \mathrm{~m}^{3} / \mathrm{s}$ avec des temps de bases d'environ 10 jours (fig. 9 et 16).

D'après les simulations, Au niveau de la Merja Fouarat le niveau des plus hautes eaux est conditionné par les écoulements des eaux débordées de l'oued Sebou dans la zone Assam. Ainsi le débit maximum au niveau de la 
jonction Assam-Fouarat est de $350 \mathrm{~m}^{3} / \mathrm{s}$ (fig.23) pour le modèle 1 et de 50 $\mathrm{m}^{3} / \mathrm{s}$ (Fig.24) pour le modèle 2 .

Les débits négatifs ont été observés dans cette jonction restent très faibles et correspondent à des écoulements de la Merja Fouarat vers la zone Assam et sont dus à la vidange de la zone Assam par le deuxième lien latéral et au blocage d'écoulement dans le canal Fouarat dont l'écoulement est bloqué par la marée.

Les lames d'eau maximales, par rapport au terrain naturel, résultant des simulations dans les zones très vulnérables situées entre Assam et Fouarat sont de $4 \mathrm{~m}$ et $2 \mathrm{~m}$ respectivement pour le modèle 1 et le modèle 2 (fig. 23 et 24). Les variations des débits et des niveaux d'eau dans les deux zones de stockage montrent qu'elles ont joué dans l'épandage des crues (fig. 25 et 26)

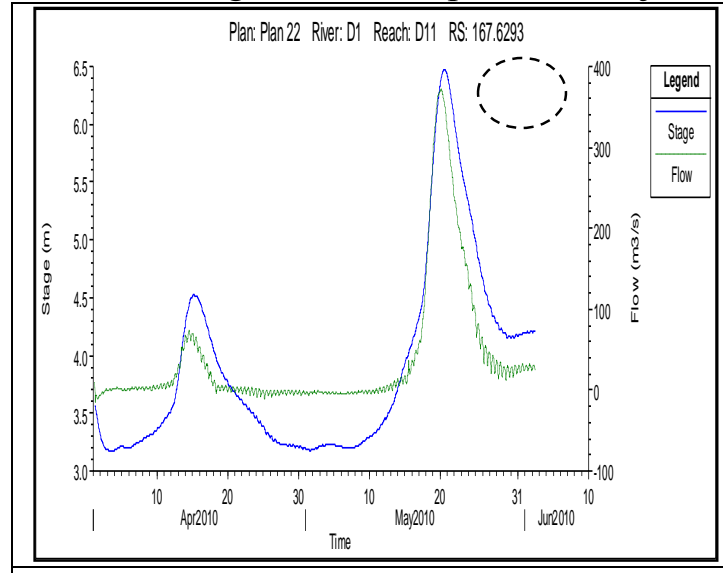

Figure 23 : Hydrogramme et niveau d'eau de la jonction entre les zones Assam et Fouarat Modèle 1

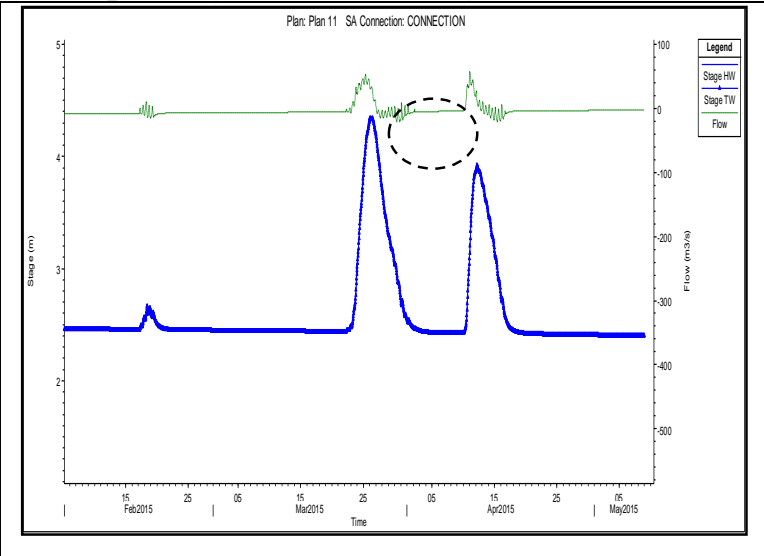

Figure 24 : Hydrogramme et niveau d'eau entre les zones Assam et Fouarat - Modèle 2

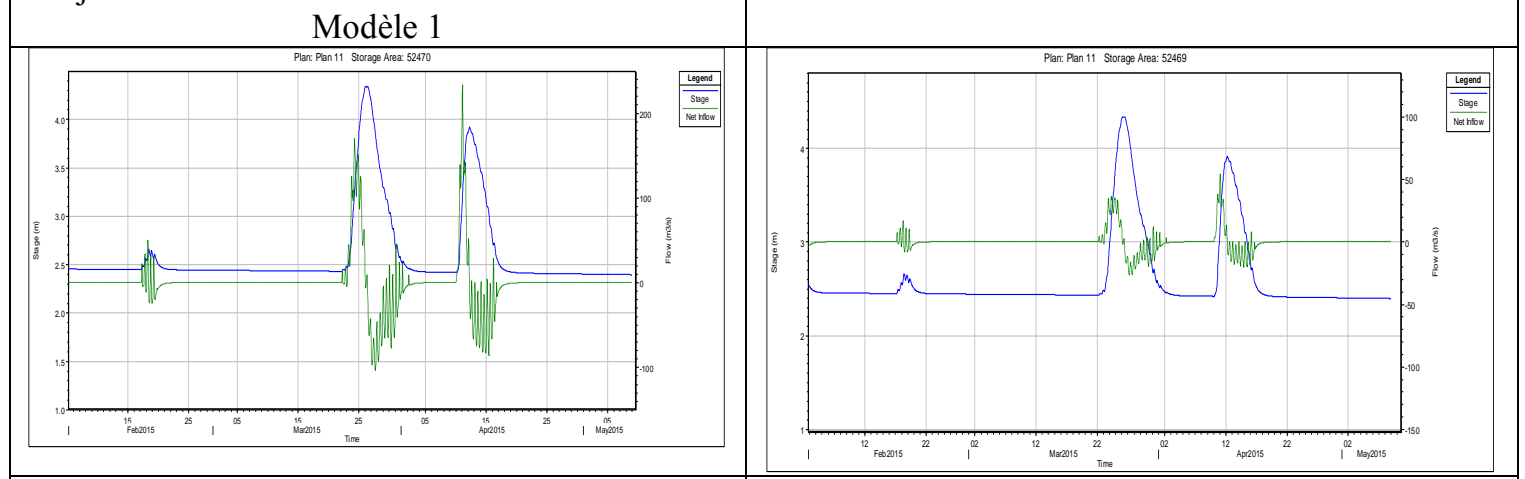

Figure 25 : Evolution du debit et du niveau d'eau dans la zone de stockage Assam Model 2

Figure 26 : Evolution du debit et du niveau d'eau dans la zone de stockage Fouarat Model 2

L'écoulement au niveau du système hydraulique Sebou-Fouarat est un écoulement fluvial présentant un nombre de Froude calculé, qui traduit le rapport des forces d'inertie aux forces de gravité, est généralement inférieur à 0,5. Ceci montre que cet écoulement, contrôlé fortement par l'aval, est due 
principalement à l'énergie potentielle de l'eau et que la part de l'énergie cinétique dans cet écoulement est très faible. En effet les vitesses maximales d'écoulement simulées pour les deux modèles sont très faibles du fait de la pente des chemins d'écoulement quasiment nulle, ces vitesses sont généralement inferieurs à $1 \mathrm{~m} / \mathrm{s}$ (fig. 27, 28, 29 et 30)

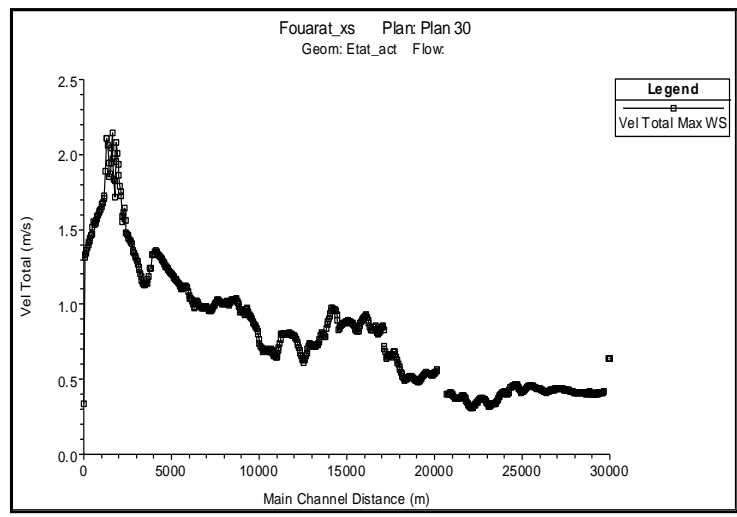

Figure 27 : Vitesses maximales dans l'oued Sebou - Modèle 1

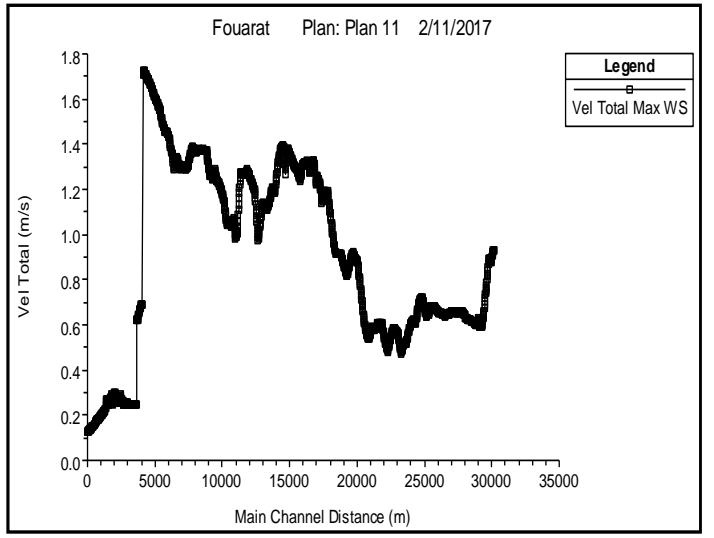

Figure 29: Vitesses maximales dans l'oued Sebou - Modèle 2

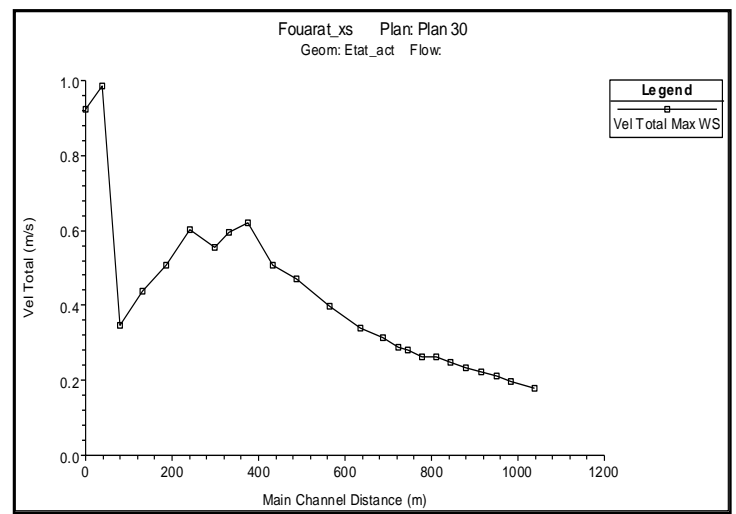

Figure 28 : Vitesses maximales dans le canal Fouarat - Modèle 1

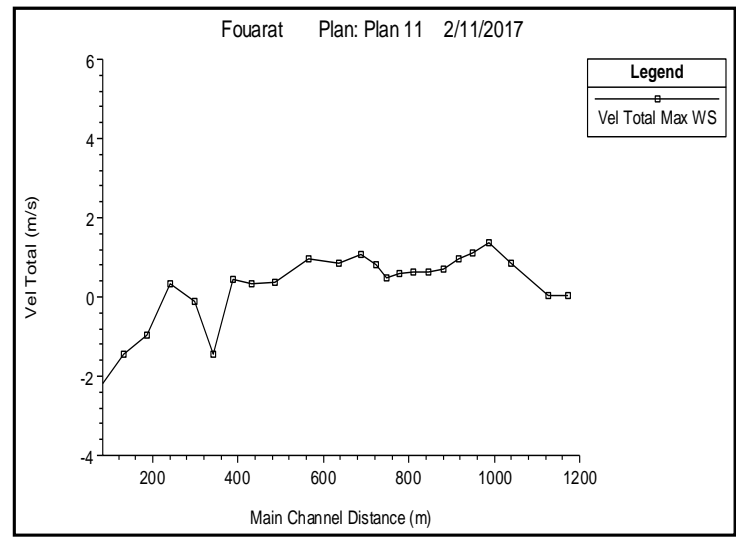

Figure 30 : Vitesses maximales dans le canal Fouarat- Modèle 2

Aux termes de propagation des crues entre le pont de l'autoroute située à l'ouest de la ville de Kenitra et la zone Fouarat, le temps de transit varie entre 10 et 12 heures et ceci à cause des faibles vitesses que présente l'écoulement.

\section{Conclusion}

Les résultats du modèle 1 sont généralement surévalués puisqu'ils donnent des niveaux des plus hautes eaux trop élevés par manque de considération des capacités importantes de stockage et d'épandage des crues. L'utilisation de ce modèle pour définir des mesures de prévention et de 
protection contre les inondations induira des surcoûts dans toute action envisageable par les décideurs.

Le modèle 2, prenant en considération les zones d'épandage de crues représente un modèle plus adéquat avec la réalité de terrain. Les résultats de ce modèle, plus plausibles et plus réalistes, peuvent constituer les bases de toutes actions visant non seulement la protection contre les inondations mais aussi la résilience avec ce risque.

Pour les deux branches Fouarat et Assam, et selon l'hypothèse pessimiste qui consiste à la concomitance entre les crues émanant de l'oued Sebou et de l'oued Fouarat, nous avons constaté que se sont les apports des débordements de l'oued Sebou qui conditionnent les inondations alors que l'effet des crues de Fouarat reste très limité.

Le risque encouru par les populations et leur bien dans la zone d'étude est lié aux hauteurs des lames d'eau calculées qui sont de l'ordre de $4 \mathrm{~m}$ au niveaux des zones stratégiques et de forte vulnérabilité dans la ville telles que la présence des quartiers à forte densité d'occupation et d'infrastructures (l'usine électrique, la route nationale et la zone industrielle).

Pour gérer les situations d'inondation au moment de la crise sachant que le temps de propagation est de l'ordre de 10 heures, il faudrait procéder à l'installation des équipements de mesure et de suivi en temps réel des niveaux d'eau sur l'oued Sebou à l'amont de la ville. Ce modèle pourrait alors être utilisé comme un outil de prise de décision par les pouvoirs publics. Ce modèle mérite également d'être développé pour prendre en considération des zones de stockage sur la rive droite de l'oued Sebou à l'aval et de simuler des plans d'aménagement à proposer pour la protection contre les inondations des urbanisées limitrophes menacées.

La prise en considération des zones tampons des Merjas Fouarat et Al Assam dans tout aménagement futur est aussi un atout pour la préservation et la restauration de ces zones humides en particulier la Merja Fouarat qui a une valeur écologique national très élevé.

\section{References:}

1. ABHS (2013). Etude de protection contre les inondations de la ville de Kenitra.

2. ABHS (2010). Etude de schéma directeur de protection de la plaine du Gharb contre les inondations.

3. Barroca, B. \& Hubert, G. (2008). Urbaniser les zones inondables, est-ce concevable ? Développement durable et territoires, Dossier 11, [En ligne] URL : http://developpementdurable.revues.org/7413 ; DOI : 10.4000/développement durable.7413 
4. Kovacs, Y. (2010). Modèles de simulation d'écoulement transitoire en réseau d'assainissement - thèse de doctorat présentée à l'école nationale des ponts et chaussées spécialité sciences et techniques de l'environnement $352 \mathrm{p}$

5. Lahrouz, S., Dakki, M., \& Gmira, N. (2011). Merja Fouarat: zone humide importante menacée par l'urbanisation - Le marécage de Fouwarat (Kénitra, Maroc) : site de conservation d'oiseaux menacé par l'urbanisation. Afrique Science, Vol. 7, N${ }^{\circ}$, ISSN 1813-548X.

6. Malaterre, P.O., Baume, J.P., Jean-Baptiste, N. \& Sau. J. (2011). Analyse pour le calage de modèles hydrauliques à surface libre : une approche par les théories des systèmes linéaires et de l'automatique. $68 \mathrm{p}$.

7. US Army Institute of Water resources (2001). Hydrologic Engineering centre - UNET One-Dimensional Unsteady Flow Through a Full Network of open chanel Users manual. 400 p. 